Proc. XIX International School of Semiconducting Compounds, Jaszowiec 1990

\title{
ON THE TUNNELING AMONG SHALLOW AND DEEP CENTERS IN ZnS *
}

\author{
A. Zakrzewski and A. Sienkiewicz
}

Institute of Physics, Polish Academy of Sciences, Al. Lotników 32/46, Warszawa, Poland (Received August 8, 1990)

\begin{abstract}
Results of the photo-ESR studies of recharging processes due to tunneling in $\mathrm{ZnS}: \mathrm{Cu}$ crystals are presented. It was found that the tunneling among shallow and deep centers seems to be a second order effect in the overall photoluminescence quenching in $\mathrm{ZnS}$ by transition metal impurities.
\end{abstract}

PACS numbers: 72.20.Jv, 76.30.Fc

\section{Introduction}

Radiative tunneling among randomly distributed donors (D) and acceptors (A) is one of the most efficient processes responsible for the visible luminescence (VL) in many semiconductors (SCs). However, the quantum yield of these transitions may be strongly affected by presence of the other, usually deep, centers. The best known deactivators (killers) of VL in $\mathrm{ZnS}$ are transition metal (TM) impurities. In the previous papers $[1,2]$ two deactivation mechanisms were identified. Recently O'Donnel et al. [3] proposed a new one which can limit the donor-acceptor pair (DAP) luminescence in $\mathrm{ZnSe}$ :Fe. During this process an electron tunnels from a donor (D) to iron instead of acceptor (A) (Fig. 1).

In this paper we present the results of photo-ESR studies of recharging process in $\mathrm{ZnS}$ crystals. By selecting the experimental temperature and conditions we could obtain the metastably occupied D, A, Fe and Cr centers with their depopulation monitored via the studies of $\mathrm{Fe}^{3+}$ and $\mathrm{Cr}^{+}$ESR signal intensities. At low temperature this depopulation occurs only due to tunneling transitions of e.g. $\mathrm{A} \Rightarrow \mathrm{Cr}$ type. Hence, the decay kinetics of the $\mathrm{Fe}^{3+}$ and $\mathrm{Cr}^{+} \mathrm{ESR}$ signals enables us to estimate the tunneling efficiencies among activator and killer centers.

The efficiency of the DAP luminescence is usually characterized by two parameters: $W_{0}$ and $\alpha$. This comes from the fact that the tunneling rate $W(r)$ between given donor and an acceptor located at a distance $r$ is taken in the form:

*This work was supported by CPBP 01.12. 
$W(r)=W_{0}^{\star} \exp (-r / \alpha)$ where $W_{0}=10^{5} \div 10^{8} \mathrm{~s}^{-1}, \alpha=8 \div 12 \AA$ for ZnS DAP emission. Hence, for easy reference, we express the tunneling rate for transitions involving $\mathrm{Fe}$ and $\mathrm{Cr}$ also by $W_{0}$ and $\alpha$. These processes may be partly or totally nonradiative, but the exponential form of $W(r)$ is generally accepted for transitions between localized states.

Such a tunneling among chromium in various charge states $\left(\mathrm{Cr}^{4+}+\mathrm{Cr}^{2+} \Rightarrow\right.$ $2 \mathrm{Cr}^{3+}$ ) was postulated earlier by Ulrici for $\mathrm{Cr}$ in $\mathrm{GaAs}$ [4], also on the basis of photo-ESR experiment. We could, thus, compare his results with our data to stress our conclusions on TM-related tunneling transitions in $\mathrm{ZnS}$.

\section{Results and Discussions}

Before the light was turned on neither $\mathrm{Fe}^{3+}$ nor $\mathrm{Cr}^{+}$ESR signals were observed. Illumination with light of $\lambda=450 \mathrm{~nm}$ neutralizes $\mathrm{D}$ and $\mathrm{A}$ centers and converts iron $\mathrm{Fe}^{2+}$ into $\mathrm{Fe}^{3+}$ and $\mathrm{Cr}^{2+}$ into $\mathrm{Cr}^{+}$. After the light was turned off a slow decay of the Fe and Cr ESR signals was observed (Fig. 2a). We explain the decay of $\mathrm{Fe}^{3+}$ and $\mathrm{Cr}^{+}$due to the tunneling: $e_{\mathrm{D}}+\mathrm{Fe}^{3+} \Rightarrow \mathrm{Fe}^{2+}$ and $h_{\mathrm{A}}+\mathrm{Cr}^{+} \Rightarrow \mathrm{Cr}^{2+}$ where $e_{\mathrm{D}}$ and $h_{\mathrm{A}}$ denote an electron localized on $\mathrm{D}$ and a hole on $\mathrm{A}$, respectively.

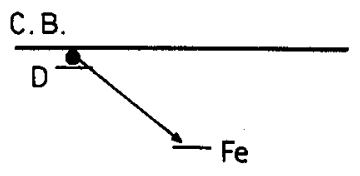

A-

V. 8 .

Fig 1. The tunneling transition between donor and iron which can compete with radiative DAP process.

Utilizing the neutrality equation: $n_{\mathrm{A}}+n_{\mathrm{Fe}}=n_{\mathrm{D}}+n_{\mathrm{Cr}}\left(n_{\mathrm{Fe}} \approx 10^{16} \mathrm{~cm}^{-3}\right.$ and $n_{\mathrm{Cr}} \approx 2 \times 10^{15} \mathrm{~cm}^{-3}$ were estimated from the ESR signal intensity) together with the fact that the sample was compensated we made the following approximation: $n_{\mathrm{A}}=n_{\mathrm{Cr}}$ and $n_{\mathrm{Fe}}=n_{\mathrm{D}}$. Under these conditions deterministic rate equations are frequently used: $\mathrm{d} n_{i} / \mathrm{d} t=-\beta n_{i}^{2}$ where $\beta$ denotes the average tunneling rate and $i$ stands for $\mathrm{D}, \mathrm{A}, \mathrm{Fe}$ or $\mathrm{Cr}$, respectively. This yields the hyperbolic solution $n_{i}(t) \sim t^{-1}$. Our kinetics, however, are not hyperbolic. This comes from the fact that the tunneling transitions are characterized by a very broad distribution of lifetimes resulting from the spread of the tunneling distances. Hence, any calculation of the decay kinetics need a very complicated averaging over all possible configurations. The deterministic approach completely ignores this averaging. For this reason, we utilize here the Monte Carlo approach proposed by Gillespie [5]. The theoretical curves were fitted to the observed kinetics (Fig. 2b). 
The following values of parameters $W_{0}$ and $\alpha$ were obtained: $W_{0}=10 \div 10^{3} \mathrm{~s}^{-1}$ and $\alpha=16 \div 19 \AA$ both for $\mathrm{Fe}$ and $\mathrm{Cr}$. We can also estimate the values of $\beta_{\mathrm{ACr}} \approx 10^{-19} \mathrm{~cm}^{3} / \mathrm{s}$ and $\beta_{\mathrm{DFe}} \approx 10^{-20} \mathrm{~cm}^{3} / \mathrm{s}$ (for initial decay) to compare them with DAP VL: $\beta_{\mathrm{DA}} \approx 10^{-13} \div 10^{-15} \mathrm{~cm}^{3} / \mathrm{s}$ [6]. For GaAs the following values are reported: $\beta_{\mathrm{DA}} \approx 10^{-9} \mathrm{~cm}^{3} / \mathrm{s}$ [7] and $\beta_{\mathrm{CrCr}} \approx 10^{-17} \mathrm{~cm}^{3} / \mathrm{s}$ [4].
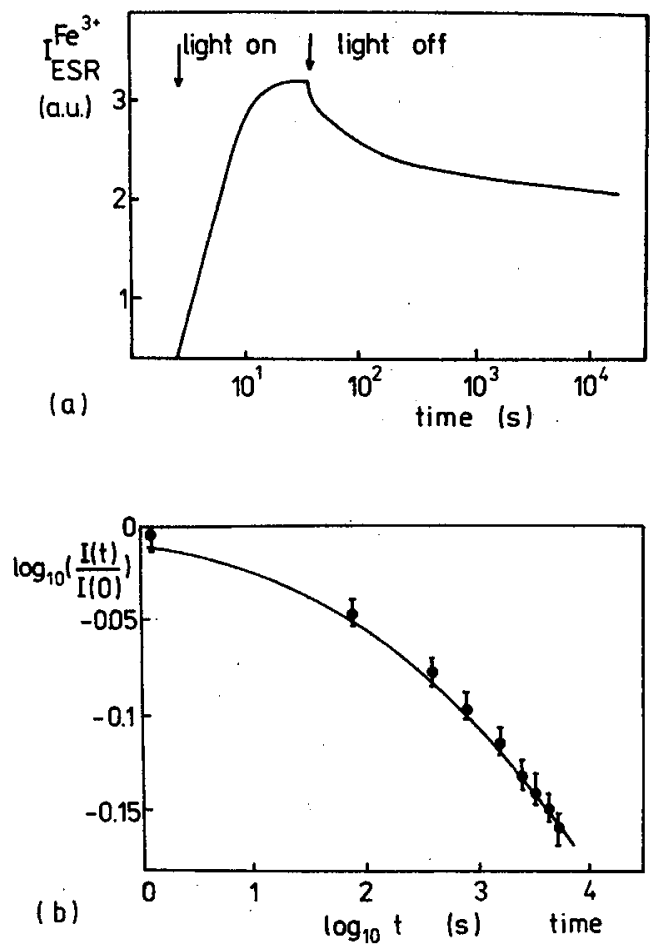

Fig. 2. a) A schematic kinetics of the $\mathrm{Fe}^{3+}$ ESR signal after turning off the exciting light ( $\lambda=450 \mathrm{~nm}, T=25 \mathrm{~K})$; b) numerical fit (solid line) to the experimental points for decay kinetics of iron $\left(W_{0}=50 \mathrm{~s}^{-1}, \alpha=17 \AA\right)$.

The obvious source for uncertainty of $W_{0}$ and $\alpha$ values is the error of the $n_{\mathrm{Fe}}$ and $n_{\mathrm{Cr}}$ estimation. The assumptions about $n_{\mathrm{D}}=n_{\mathrm{Fe}}$ and $n_{\mathrm{A}}=n_{\mathrm{Cr}}$ is less critical. Comparing these values with those for radiative transitions one can conclude that the tunneling seems to be a second order effect in the overall VL reduction in $\mathrm{ZnS}$ based phosphors by TM impurities. It should be stressed here that in our experiments only distant pairs D-Fe and $\mathrm{A}-\mathrm{Cr}$ are studied. Hence, our conclusions are valid only if correlation effects are negligible.

\section{References}

[1] A. Zakrzewski, M. Godlewski, Phys. Rev. B 34, 8993 (1986). 
[2] A. Zakrzewski, M. Godlewski, J. Appl. Phys. 67, 2457 (1990).

[3] K.P. O'Donnell, K.M. Lee, G.D. Watkins, J. Phys. C 16, L723 (1983).

[4] W. Ulrici, Phys. Status Solidi B 131, 707 (1985).

[5] D.T. Gillespie, J. Comput. Phys. 22, 403 (1976).

[6] H. Przybylińska, M. Godlewski, Phys. Rev. B 36, 1677 (1987).

[7] J. Zeiger, J. Appl. Phys. 35, 1657 (1964). 